\title{
The Effectiveness Of Aerobic Exercise In Improving Functional Status : A Case Report
}

\author{
Carissa Putri Crisdayani ${ }^{1}$, Tresia Fransiska Tambunan ${ }^{1}$
}

1. Department of Physical Medicine and Rehabilitation, PGI Cikini Hospital, Jakarta, caíissa.putíi.cíisdayani7@gmail.com

2. Department of Physical Medicine and Rehabilitation, PGI Cikini Hospital, Jakarta

\section{Case Diagnosis:}

Chronic obstructive pulmonary disease (COPD) is a lung disease that causes ventilation abnormalities of respiratory tract obstruction that is progressive and not fully reversible. The disease is associated with a switch from a self•limiting inflammatory response, mainly initiated by smoke inhalation, to a chronic persistent inflammatory response after prolonged interaction with cigarette smoke. The pathological mechanisms and clinical manifestations of COPD are not restricted only to pulmonary inflammation and airway remodeling. The known systemic manifestations of COPD include systemic inflammation, cardiovascular comorbidities, and muscle dysfunction, leading to the decreased functional status of COPD. Chronic inflammation in the pulmonary tissue is also associated with systemic effects. Systemic inflammation may then lead to skeletal muscle atrophy and cachexia. It may also initiate and worsen comorbidities. ${ }^{1}$ It limits the patient's exercise capacity and the ability to perform activities of daily living (ADL), leading to a reduced quality of life. Quantification of physical ADL revealed that COPD patients spend less time standing and walking daily than healthy elderly individuals. ${ }^{2}$ Pulmonary rehabilitation is a comprehensive, multidisciplinary intervention designed to reduce symptoms, increase functional status and quality of life, and focus on the primary and secondary impairments associated with respiratory disease in COPD patients. Strategies include exercise training, self-management education, nutritional intervention and psychosocial support. $^{3}$

Keywords: Aerobic Exercise, Functional Status, Chronic obstructive pulmonary disease

Mr. D, a 76year-old-man with COPD GOLD 2 Group B was consulted with complaints of fatigue in daily activities. One of his daily activities was walking to the mosque, which was approximately 500 meters away. Symptoms began in the past 2 years and had progressively worsened with no known associating, aggravating, or relieving factors. Before the exercise started, the 6-minute walk test was conducted to obtain the predicted track length and exercise prescription. The predicted distance for this patient was $578.4 \mathrm{~m}$, and the actual distance before the first exercise was $346.5 \mathrm{~m} \mathrm{(59 \% ).} \mathrm{The}$ interpretation of the first distance percentage was very poor. Before the pulmonary rehabilitation exercise began, the patient's CAT score was 30 . The patient was given an aerobic exercise on the treadmill with a speed of $1.74 \mathrm{~km} / \mathrm{h}$ (moderate-intensity or $60 \%$ ) three times a week for 8 weeks. After 8 weeks of exercise, there was an increase of 6-minute walk distance from 346.5 to 476 $\mathrm{m}$. There was also an improvement in clinical symptoms with a decreased CAT score from 30 to 21 .

Table 1. Result of 6 Minute Walking Test and CAT score 


\begin{tabular}{|c|c|c|c|}
\hline METS & 4.05 & 4.36 & 4.78 \\
\hline Predicted distance $(\mathrm{cm})$ & 578.41 & 578.41 & 578.41 \\
\hline Actual distance (cm) & 346.5 & 402 & 476 \\
\hline Percentage & 59 & 69 & 82 \\
\hline CAT score & 30 & - & 21 \\
\hline
\end{tabular}

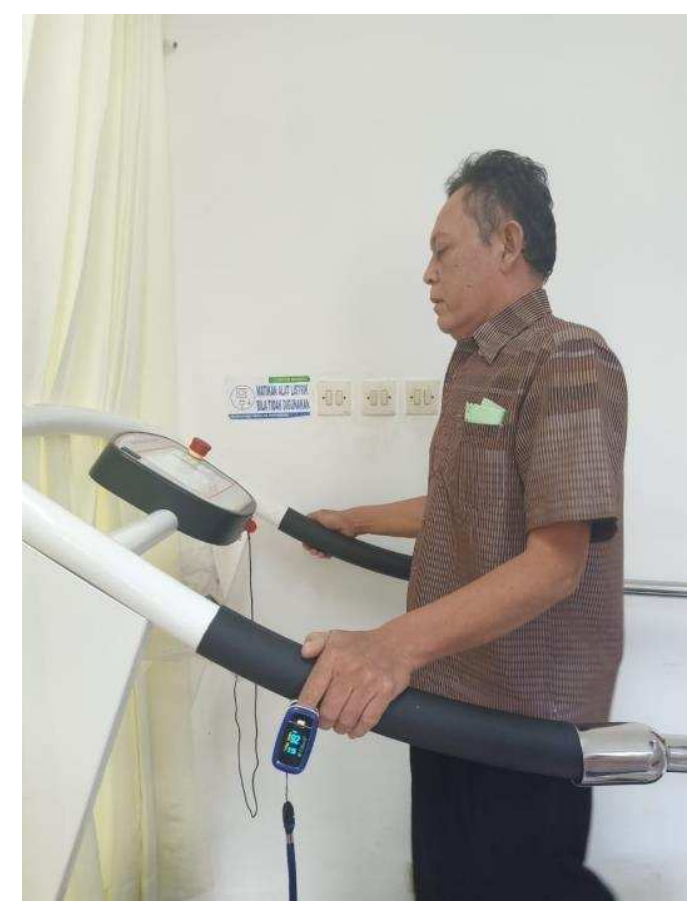

Picture 1. Mr. D was given a moderate-intensity aerobic (treadmill) exercise

Discussion : Exercise training performed in the context of a pulmonary rehabilitation program has been shown to be effective in several outcomes of patients with COPD, such as improved exercise tolerance, muscle strength, quality of life, and reduced dyspnea and fatigue. ${ }^{3}$ The 6minute-walk distance (6MWD) has proven to be useful in assessing the functional status of patients with COPD. The threshold for the minimum clinically important difference (MCID) in recent estimates is $25-35 \mathrm{~m}$. This range should now be considered to represent a clinically important change for the 6MWD in people with COPD. ${ }^{4}$ CAT scores from 21-30 indicate a high impact of COPD on a patient's life, as shown at admission. The average CAT score at discharge turned out to be a medium impact, as a defined range from 10-20 points. The minimal clinically important difference (MCID) for CAT is supposed to be a change of 2 points. ${ }^{5}$ After 8 weeks of exercise, there was an increase in the patient's 6MWD distance and improved clinical symptoms with a decreased CAT score.

Conclusion:

Comprehensive cardiopulmonary rehabilitation for COPD patients can improve the patient's functional capacity and quality of life, which was reflected in the patient's functional ability to perform his daily activities.

1. Barnes PJ, Celli BR. Systemic manifestations and comorbidities of COPD. Eur Respir J. 2009;33:1165-85. 
2. Lahaije A, van Helvoort H, Dekhuijzen PNR, Heijdra YF. Physiologic limitations during daily life activities in COPD patients. Respir Med. 2010;104:1152-9.

3. Gloeck1 R, Marinov B, Pitta F. Practical recommendations for exercise training in patients with COPD. Eur Respir Rev. 2013;22:178-86.

4. Holland AE, Nici L. The return of the minimum clinically important difference for 6minute-walk distance in chronic obstructive pulmonary disease. Vol. 187, American Journal of Respiratory and Critical Care Medicine. 2013. hal. 335-6.

5. Schultz K, Gohl O, Stojanovic D, Witmann M, Rudinik J, Schawarze M. CAT (COPD assessment test) as outcome parameter of pulmonary rehabilitation (PR) in COPD. Eur Respir J. 2011;38:3626. 